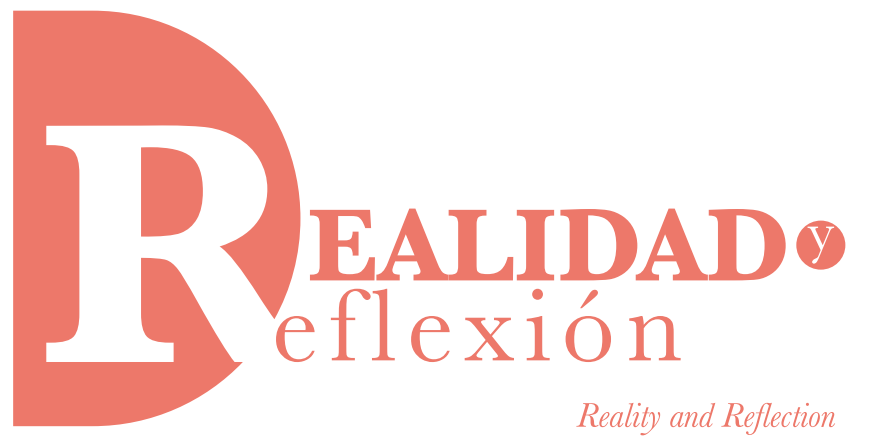

ISSN 1992-6510

e-ISSN 2520-9299

Año 19, N 49, San Salvador, El Salvador, Centroamérica. Revista Semestral Enero-Junio 2019

YEAR 19, N 49, SAN SALVADOR, El SALVADor, CENTRAL AMERICA. SEMESTRAL JoURNAL JANUARY-JUNE 2019

\title{
Agricultura orgánica, maíz criollo y extensión rural: percepciones de profesionales salvadoreños
}

\section{Organic agriculture, maize and rural extension: perception of Salvadorian professionals}

\author{
Carlos Ayala Durán (Autor principal) \\ Universidade Federal do Rio Grande do Sul (UFRGS) y Universidad de El Salvador. \\ Doctor en Desarrollo Rural por la Facultad de Ciencias Económicas de la Universidade Federal do Rio Grande do Sul, Brasil. \\ carlos.ayala@ufrgs.br \\ Paulo Dabdab Waquil \\ Universidade Federal do Rio Grande do Sul (UFRGS). Programa de Pós Graduação em Desenvolvimento Rural. Agrónomo \\ Doctor en Economía Agrícola por la University of Wisconsin-Madison (Estados Unidos). \\ waquil@ufrgs.br
}

\section{RESUMEN}

El presente artículo tiene como objetivo realizar un análisis exploratorio y cualitativo sobre las percepciones de extensionistas rurales públicos salvadoreños en relación a la agricultura orgánica y a la producción de maíz utilizando semilla criolla. Para tal fin, fueron encuestados todos los extensionistas públicos del Centro Nacional de Tecnología Agropecuaria y Forestal (CENTA) laborando en los departamentos de La Libertad y Sonsonate ( $\mathrm{N}=28)$. Los resultados muestran que los extensionistas encuestados consideran a la agricultura convencional como más lucrativa y menos amigable con el medio ambiente, en contraposición a la agricultura orgánica. De la misma forma, estos profesionales del sector público consideran al maíz criollo en términos generales como menos productivo y lucrativo que el maíz híbrido, pese a que el primero es visto como mejor adaptado a las condiciones ambientales y climáticas del país.

Palabras clave: Palabras clave: maíz, El Salvador, orgánico, CENTA, extensión, criollo.

\section{ABSTRACT}

The following study has the objective to perform an initial, exploratory and qualitative analysis on the perceptions of public rural extensionists in El Salvador regarding organic agriculture and maize production using creole (native) seed. To do so, all public extensionists from the official agency National Centre of Agricultural and Forest Technology (CENTA) working in the states of La 
Libertad and Sonsonate $(N=28)$ were surveyed. Results show that extensionists considered conventional agriculture as more lucrative and less environmentally friendly than organic agriculture. Likewise, these professionals considered that creole maize is less productive and less lucrative than hybrid maize, though native seed is seen as better adapted to national climate and environmental conditions.

Keywords: maize, El Salvador, organics, CENTA, extension, creole.

\section{Introducción}

Actualmente se evidencia cómo las temáticas socioambientales ganan mayor importancia a nivel mundial. De esa forma, temas como el aumento poblacional, cambio climático, disminución de la tierra cultivable, seguridad alimentaria, desertificación de tierras, contaminación asociada a la agricultura y encarecimiento de los combustibles fósiles (Gomiero, Paoletti y Pimentel, 2008) han desbordado la arena técnica-científica, y se han desparramado hasta permear arenas gubernamentales, políticas y comunicacionales. De esa forma, Wheeler (2008a) señala cómo a nivel mundial, la opinión pública ha mostrado un creciente interés en relación al bienestar ambiental y de los agroecosistemas. Esto genera por su vez un aumento en los esfuerzos de regulación agrícola así como un incremento de iniciativas que incentivan a que los agricultores adopten prácticas agrícolas más sustentables (Chang y Kristiansen, 2006). Una de las prácticas que suelen considerarse más amigables con el medio ambiente es la agricultura orgánica. Según la Organización de las Naciones Unidas para la Alimentación y la Agricultura (2004), la agricultura orgánica y el manejo sustentable de bosques, además de producir comodities, facilitan la construcción de sistemas alimentarios auto-regenerativos. De la misma forma, se considera que el aumento de estas prácticas agrícolas sustentables pueden representar una política eficiente para promover la biodiversidad (FAO, 2004).

Particularmente en El Salvador, la agricultura orgánica presenta una utilización marginal en la agricultura a nivel nacional. De esta forma, el último censo agropecuario realizado en el país retrata por ejemplo que el 89.9\% de los agricultores a nivel nacional utilizan control químico de plagas (Ministerio de Economía de El Salvador [MINEC], 2009). De forma similar, este censo retrata únicamente 717 hectáreas plantadas con orgánicos (MINEC, 2009). Pese a que existe evidencia sobre los beneficios económicos, ambientales y de salud en relación a la producción orgánica, (Bengtsson, Ahnström, y Weibull, 2005; Nelson et al., 2004, Mäder et al., 2002; O'Riordan y Cobb, 2001; Stolze et al., 2000, Wynen, 1988; Wives, 2008) existen profesionales que dudan de la viabilidad financiera de esta práctica (Wheeler, 2008b). De esta forma, las actitudes negativas o conservadoras de la comunidad científica y académica se erigen como barreras para la investigación, extensión y adopción de la agricultura orgánica por parte de agricultores (Wheeler, 2008b; Larson y Duram, 2000; Duram y Larson, 2001).

En virtud que El Salvador tiene una tradición milenar con relación a la producción y consumo del maíz, otra de las prácticas agrícolas que suele argumentarse tienen menor impacto ambiental es la producción de maíz con semilla criolla. 
No obstante, como producto del incremento de tecnologías asociadas a la revolución verde desde la mitad del siglo pasado, la cultura de este cereal ha visto sus prácticas modificadas, reduciéndose la importancia del maíz criollo en la producción nacional. Adicional a la utilización de insumos químicos en la agricultura salvadoreña, se ha potenciado tanto desde la arena pública como la privada, la paulatina substitución de semillas criollas originarias, por semillas mejoradas, destacadamente las semillas de origen híbrido. De tal forma, el último censo agropecuario refleja que en todo el país, el 79.05\% del total del área plantada con maíz fue realizada con semilla híbrida (MINEC, 2009). Este nivel de penetración de las semillas híbridas contrasta con otros países que tienen larga tradición en el cultivo del maíz, siendo que por ejemplo México obtiene más de un $80 \%$ de su producción utilizando semilla criolla, reduciéndose a tan sólo 18.8\% la producción con semilla híbrida (United States Department of Agriculture- Secretaria de Agricultura Ganaderia y Desarrollo Rural [USDA-SAGAR],1997 apudMAGDALENOHERNÁNDEZ et. al., 2016).

Adicionalmente, este cambio en la utilización del paquete tecnológico en la cultura del maíz en El Salvador tendrá aparejado unos costos de producción altos. De esa forma, Martínez (2007) indica que en la producción de maíz en El Salvador, el costo de los agroquímicos asciende al $33.7 \%$ del total. Paralelamente, la adquisición de semilla implica un 4.6\%. De tal suerte, casi el 40\% del costo total para producir maíz en El Salvador es atribuible únicamente a estos dos insumos. Tal gasto representa un alto costo que debe ser asumido por los agricultores salvadoreños, y como se ha evidenciado en el año 2008, el precio final de los alimentos puede aumentar sustancialmente en virtud del encarecimiento de los insumos derivados del petróleo (Banco Central de Reserva [BCR], 2008).

El aumento en la utilización de insumos químicos en la agricultura en términos generales, y la utilización de semilla híbrida para el cultivo de maíz en El Salvador, ha venido catalizado por el notable apoyo público a este tipo de tecnología. Uno de los primeros pasos fue la legalización de estas prácticas en la década de los años setenta, mediante la promulgación de la Ley de Certificación de Semillas y Plantas (1971) seguida de Ley sobre Control de Pesticidas, Fertilizantes y Productos para Uso Agropecuario (1973). Paralelamente, desde el Centro Nacional de Tecnología Agropecuaria y Forestal (CENTA), órgano público especializado en investigación y extensión rural, se ha promovido históricamente la investigación, desarrollo y validación de semillas híbridas de maíz. En ese sentido, actualmente el mencionado centro público realiza la producción de progenitores del maíz híbrido H-59, para ser provisto a organizaciones privadas con fines comerciales, ya que estos últimos entes son los encargados exclusivos de la comercialización de los diferentes tipos de maíz a nivel nacional. ${ }^{1}$ Similarmente, en tiempos más recientes, la semilla $\mathrm{H}-59$ ha sido incluida en los paquetes agrícolas que el gobierno dona a agricultores, como forma de apoyar la producción de granos básicos. Finalmente, los órganos de extensión rural públicos son usualmente

1 El Estado no vende directamente semilla a particulares. De tal suerte, se da la contradicción que CENTA produce los progenitores de maíz H-59, lo vende a entes privados para su reproducción, y finalmente compra el producto final para ser donado a agricultores parte del paquete de apoyo al cultivo de granos básicos. 
anuentes a la utilización de semilla híbrida a nivel nacional, llegando incluso a recomendar su utilización a los agricultores a los que les proveen de asesoría técnica.

Pese a que existe una notada predominancia en la utilización de técnicas oriundas de la revolución verde en la agricultura salvadoreña en general, y en la cultura del maíz en particular, comienzan a hacerse cada vez más patentes iniciativas públicas que promueven una agricultura ambientalmente más responsable. En tal sentido, dentro del CENTA existe el Banco de Germoplasma, que colecta y almacena materiales genéticos criollos de una variedad de cultivos, incluyendo maíz. De forma paralela, se ha desarrollado recientemente un proyecto a nivel nacional para la utilización de biofertilizantes. ${ }^{2}$ Esta iniciativa provee y promociona la utilización de biofertilizantes en el cultivo de maíz, frijol y sorgo; como forma de reducir el deterioro ambiental y la pérdida de rentabilidad (Centro Nacional de Tecnología Agropecuaria y Forestal [CENTA], 2015). En menor escala, en el municipio de Ciudad Arce (zona central del país), se ha realizado desde 2016 un proyecto para el cultivo de hortalizas orgánicas en invernaderos, financiado por el Ministerio de Agricultura y Ganadería (Ministerio de Agricultura y Ganadería [MAG], 2016). A este impulso público habría que sumarle el creciente interés de actores privados e intragubernamentales en temas relacionados. De esa forma, se identifica la existencia de un Programa de Rescate de Variedades de Maíz Criollo (Moreno, 2000) o la elaboración de

2 El proyecto Biofertilizantes en Cultivo de Maíz, Frijol y café como Alternativa Agroecológica para una Producción Sostenible en E1 Salvador inició su ejecución en 2015, con previsión de finalización en Noviembre de 2018 y con un presupuesto asignado de $\$ 1.5$ millones de dólares. estudios sobre como producción y conservación de semillas criollas (Asociación Fundación para la Cooperación y el Desarrollo Comunal de E1 Salvador [CORDES], 2016; Rivas et al., 2013).

Pese a que existen cada vez más iniciativas a nivel nacional y regional que buscan realizar prácticas como la agricultura orgánica o la producción de maíz con semilla criolla, parece existir una brecha en la información técnica en la utilización de este tipo de tecnología. Según Van den Ban y Hawkings (1988), las agencias públicas responsables por temas agrícolas y su personal son una de las principales fuentes de información para los agricultores. Pese a ello, en Latinoamérica la investigación en temas de extensión rural no ha sido el foco de los estudios socio agrarios (Méndez, 2006), existiendo una particular carencia en relación a las visiones de los extensionistas rurales, profesionales que suelen tener el primer contacto directo con agricultores.

A pesar de esta relativa carencia, es posible identificar algunos trabajos que desde la década de los años noventa estudiaban a los órganos públicos de extensión rural y sus extensionistas en América Latina. En ese sentido, Goodell, Andrews y López (1990) centraban su atención en el papel agrónomo-antropólogos en tareas de extensión rural en Honduras. En el sur de Brasil, trabajos como los de Zilli, Bregt y Rossiter (2006) o Hötzel y Sneddon (2013) resultan pertinentes. Los primeros investigaron las percepciones de riesgo de extensionistas rurales y agricultores, en cuanto los segundos se centraron en el papel de los extensionistas rurales en la aplicación de prácticas de alivio del dolor al descornar novillos. En el vecino país Uruguay, Gravina (2016) se centró en el 
liderazgo de los extensionistas rurales, así como la importancia del liderazgo para la ejecución de su trabajo de extensionistas. Más recientemente, diversos trabajos realizados por Fernando Landini han investigado a los extensionistas rurales de diversos países latinoamericanos como Uruguay (Landini y Riet, 2015), Brasil (Landini, 2015), Ecuador (Landini y Bianqui, 2014b) Paraguay (Landini, 2012; Landini, Bianqui y Crespi, 2013) Argentina (Landini, 2016b; 2016d), E1 Salvador (Landini, 2015b); así como Latinoamérica en su conjunto (Landini y Bianqui, 2014; Landini, 2016; 2016c). El autor realiza sus investigaciones principalmente desde la psicología, particularmente centrándose en las sinergias que pueden existir entre la psicología y la extensión rural.

El creciente interés en este tipo de investigaciones sobre extensión rural, y particularmente aquellas que abordan las visiones sobre los extensionistas rurales, parece no haber permeado aún en El Salvador. De esa forma, fue posible identificar tan solo un estudio que aborda exclusivamente las percepciones de extensionistas rurales en este país (Landini, 2015b). En el mencionado trabajo, Landini (2015b) se centraba en los problemas enfrentados por extensionistas públicos salvadoreños, queriendo a su vez indagar cuál era su concepción en la extensión rural. Únicamente de forma tangencial, los extensionistas salvadoreños son sujeto de investigación en estudios como los de Ardila (2010), Escobar (2012), Landini y Bianqui (2014) y Landini (2016, 2016c); ya que los profesionales salvadoreños son estudiados de forma simultánea a sus pares oriundos de diversos países de América Latina. En ese sentido, no fue posible identificar mayores investigaciones que se centrasen exclusivamente sobre extensionistas rurales salvadoreños, particularmente en relación a su visión sobre prácticas más sustentables.

De esta forma, en virtud que las agencias públicas responsables por temas agrícolas y su personal son una de las principales fuentes de información para los agricultores (Van den Ban y Hawkings, 1988), al papel marginal al cual ha sido relegada la agricultura orgánica (MINEC, 2009), a la importancia fundamental que reviste el maíz (y la tortilla de maíz) en la ingesta diaria de los salvadoreños (Menchú y Méndez, 2011; Ayala, 2018), a que existe en términos generales una baja utilización de tecnologías ambientalmente más limpias y a la ausencia de numerosas investigaciones que tomen en consideración a los extensionistas rurales salvadoreños; el presente trabajo tiene como objetivo analizar la visión de extensionistas públicos rurales en El Salvador, específicamente en lo que concierne a dos temas: la agricultura orgánica y la producción de maíz con semilla criolla. El presente artículo es un trabajo exploratorio necesario para comenzar a entender la visión de estos funcionarios públicos, buscando sentar bases para entender su influencia en la alta utilización de insumos químicos y la cultura de maíz con semilla híbrida a nivel nacional. Para tal fin, a continuación se definen cuáles fueron los materiales y métodos utilizados para el siguiente artículo. Posteriormente se destacan los resultados y elabora su discusión. Finalmente, se aborda las conclusiones.

\section{Metodología}

El presente trabajo forma parte de una investigación mayor, donde se combinan metodologías cuantitativas y cualitativas para predecir los factores que influyen en la 
recomendación para el uso de determinadas prácticas agrícolas por parte de los extensionistas rurales. El caso específico del presente trabajo, es de naturaleza exploratoria y descriptiva, utilizando una de las agencias de CENTA en La Libertad y Sonsonate. Estos cuestionarios contaban en primer lugar con preguntas de información demográfica para caracterizar a los extensionistas.

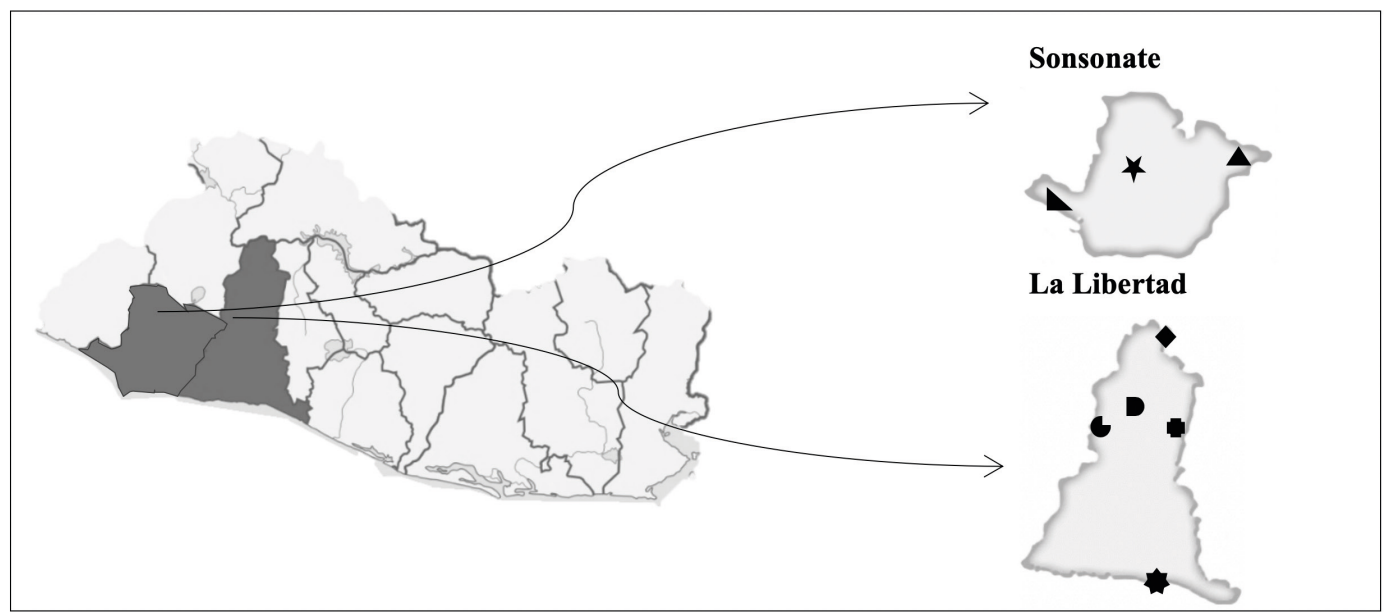

Símbolos: $\mathbf{A}$ El peñón, $¥$ Sonsonate, $\Delta$ Armenia, $\bullet$ Atiocoyo, $\triangleright$ San Juán Opico, $\bullet$ Zapotitán, $\bullet$ Quezaltepeque, - Puerto la Libertad.

Figura n. ${ }^{\circ}$ 1. Ubicación de las agencias de extensión de CENTA en La Libertad y Sonsonate (en gris). Fuente: Elaboración de los autores.

únicamente técnicas cualitativas para su desarrollo. Para el mismo, se tomaron en consideración a todos los extensionistas públicos rurales que trabajan en las diferentes Agencias de Extensión del Centro Nacional de Tecnología Agrícola y Forestal (CENTA) en los departamentos de La Libertad y Sonsonate ( $\mathrm{N}=28)$, según el mapa 1. En total existen cinco agencias de extensión rural en La Libertad (Atiocoyo, Quezaltepeque, Puerto de la Libertad, San Juán Opico y Zapotitán) y tres en Sonsonate (Armenia, El Peñón, Sonsonate).

El trabajo de campo se realizó en dos instancias. En primerlugar,serealizaron cuestionariossemiabiertos que fueron respondidos individualmente por todos los extensionistas públicos asignados a cada
Posteriormente, se indagaba la percepción de estos funcionarios en relación a la agricultura orgánica y a la producción de maíz con semilla híbrida/criolla mediante preguntas semi abiertas. A seguir, las visiones de los extensionistas en estos dos temas eran exploradas utilizando una escala Likert (15) con diversas afirmaciones. En esta escala se ponderaban diversas afirmaciones tratando temas de agricultura orgánica y cultura de maíz criolla / hỉbrida, siendo que el valor 1 indicaría estar en total desacuerdo con la afirmación, mientras que el valor 5 indicaría estar totalmente de acuerdo. Los resultados de la escala Likert fueron tabulados en el programa SPSS 23, reportando en el presente texto los valores correspondientes a estadísticas descriptivas. Las mencionadas afirmaciones eran 
basadas en estudios previos sobre agricultura orgánica (Wheeler, 2008b) y selección de maíz criollo (Madgaleno-Hernández et al., 2016; Cortez,2014); así como en posibles motivos que los extensionistas pueden concebir como decisivos en su valoración de este tipo de tecnología. Se decidió realizar las preguntas abiertas en primer lugar y después utilizar la escala Likert con el objetivo que las afirmaciones contenidas en la mencionada escala no influenciaran las respuestas abiertas de los extensionistas. En básicamente todos los casos, los cuestionarios fueron respondidos de forma presencial en la propia agencia de extensión del funcionario público, con la única excepción de los respondientes de la agencia Quezaltepeque, que enviaron el cuestionario vía e-mail. En las siete agencias restantes, posterior al llenado de los cuestionarios, se realizaban reuniones con todos los extensionistas donde se discutía cuestiones relativas a su conocimiento sobre agricultura orgánica y maíz criollo, con un doble fin: Obtener información cualitativa que pudiese no reflejarse en el cuestionario y para obtener retroalimentación de las opiniones expresadas en ese instrumento. Este trabajo de campo se realizó entre los meses de marzo 2017 hasta febrero de 2018.

Por otra parte, el presente documento tiene como marco teórico general los sistemas agroalimentarios localizados (SIAL). Los mismos fueron originalmente definidos como "Organizaciones de producción y servicios (unidades de producción agrícola, empresas agroalimentarias, comerciales, de servicio, gastronómicas...) asociadas por sus características y funcionamiento a un territorio específico" (CIRAD, 1996, p. 5). Para el abordaje de los sistemas agroalimentarios localizados, existen algunas metodologías elaboradas principalmente por académicos franceses y latinoamericanos. Entre ellas se puede mencionar las elaboradas por Malafaia y Barcellos (2007) o Grass, Cervantes y Palacios (2016). No obstante, una de las primeras metodologías desarrolladas y que mayor repercusión ha tenido en las investigaciones que utilizan SIAL como referencial teórico es la desarrollada por Muchnik(2006). En ella, el autor francoargentino estructuró los SIAL en torno a cuatro dimensiones: Histórica, técnica, institucional y alimenticia. Estas dimensiones abarcarían según el autor (Muchnik, 2006):

A. Dimensión histórica: permitiría apreciar el proceso de la formación de experiencias estudiadas. Para Ambrosini, Filippi y Miguel (2008), el sentimiento de pertenecer y la valorización de identidades formadas por una historia común podrían encajarse dentro de la dimensión histórica.

B. Dimensión técnica: estaría enfocada en la observación, descripción y análisis de los saberesy técnicasutilizadas. En esta dimensión estaría incluido el know-how o saber hacer (Ambrosini; Filippi; Miguel, 2008; Specht, 2009) para el cultivo o transformación de los productos agroalimentarios.

C. Dimensión alimenticia: estaría preocupada con las relaciones (sociales, culturales, económicas) entre el producto y el consumidor. De esa forma, los productos de un SIAL se diferencian por características intrínsecas propias de un ambiente determinado (Specht, 2009).

D. Dimensión institucional: tendría como foco las relaciones entre los actores sociales y sus estrategias individualesy/o colectivas(Muchnik, 2006). Esta dimensión podría abarcar arreglos entre productores, formación de cooperativas, 
sistemas de compras, actuaciones de los poderes públicos, entre otras.

De forma paralela a estas cuatro dimensiones originalmente formuladas por Muchnik (2006), el autor propuso una modelización cualitativa, utilizando cuatro objetos de investigación, a saber: Coordinación de los actores, cualificación de los productos, gestión de los recursos y dinámica de los saberes y competencias. (Muchnik, 2006, 2012). No obstante, se evidencia que los trabajos utilizando SIAL son mayormente empleadas las mencionadas dimensiones (Specht, 2009, 2014; Chechi; Schulzy Niederle, 2016; Ambrosini, Filippi y Miguel, 2008, 2009). De esa forma, tomando así en consideración estas cuatro dimensiones, el siguiente trabajo se centra en la dimensión institucional del SIAL, y busca focalizarse en uno de los actores públicos más visibles en el campo y menos estudiados: Los extensionistas públicos rurales. De tal suerte, Conocer la opinión de estos servidores públicos sobre el uso de estas tecnologías resulta fundamental para pensar en estrategias a futuro que vayan en

Tabla n..$^{\circ} 1$

Información general sobre personal de agencias públicas de extensión y número de agricultores censados por municipio

\begin{tabular}{c|c|c|c}
\hline Agencia de Extensión & $\begin{array}{c}\text { Municipio donde se } \\
\text { localiza }\end{array}$ & $\begin{array}{c}\text { Número de extensionistas } \\
\text { en agencia }\end{array}$ & $\begin{array}{c}\text { Número de agricultores } \\
\text { según último censo. }\end{array}$ \\
\hline Atiocoyo & San Pablo Tacachico & 4 & 2.677 \\
\hline Quezaltepeque & Quezaltepeque & 3 & 2.344 \\
\hline Puerto la Libertad & La Libertad & 3 & 2.427 \\
\hline San Juán Opico & San Juán Opico & 5 & 6.341 \\
\hline Zapotitán & Ciudad Arce & 5 & 4.301 \\
\hline Armenia & Armenia & 2 & 2.575 \\
\hline El Peñón & Acajutla & 2 & 3.341 \\
\hline Sonsonate & Sonsonate & 4 & 3.379 \\
\hline
\end{tabular}

contracorriente, como los mencionados huertos orgánicos (MAG, 2016) o los programas de biofertilizantes (CENTA, 2015). En ese sentido, Wheeler (2008b) manifiesta que el tener acceso a información fidedigna y relevante se considera como un componente crítico para influenciar a que los agricultores decidan adoptar prácticas alternativas, nuevas tecnologías e innovaciones.

\section{Resultados y discusión}

En primer lugar, es destacable que el número total de extensionistas en estos dos departamentos es bastante limitado. Si se toma en consideración únicamente el número de agricultores que existía en los municipios donde se encontraban localizadas estas agencias de extensión, se evidencia un número limitado de extensionistas por agencia, según lo muestra la tabla 1 . De esta forma, los 28 extensionistas públicos tendrían teóricamente que atender un público meta de 27.385 agricultores. A esto habría que adicionar el hecho que en diversas ocasiones, las agencias de extensión dan asesoría a agricultores que se

\footnotetext{
Fuente: Elaboración propia en base a MINEC (2009)
} 
localizan en municipios aledaños. Por ejemplo, la Agencia Zapotitán, localizada en Ciudad Arce, da cobertura a agricultores del municipio de Colón y Comasagua. Por su parte, la agencia Atiocoyo, situada en San Pablo Tacachico, atiende a agricultores de los municipios vecinos E1 Paisnal, Guazapa y Aguilares. Según fuentes consultadas, este déficit parece ser explicado por restricciones presupuestarias. En tal sentido, desde la segunda mitad del siglo XX se reporta una reducción constante del presupuesto público agrícola en El Salvador (Ardila, 2010; Fundación Salvadoreña para la Reconstrucción y el Desarrollo [REDES], 2014).

Los extensionistas rurales de estos dos departamentos tienen una edad media de 49.57 años $(\sigma=11.01)$, con 22.3 años en media de experiencia laboral $(\sigma=10.68)$. La gran mayoría de ellos son hombres, habiendo únicamente entre sus filas cuatro mujeres, lo que implicaría alrededor de un $14 \%$ de los extensionistas públicos correspondientes al sexo femenino. E1 estudio realizado por Landini y Bianqui (2014) retratan leves diferencias a los extensionistas públicos salvadoreños sujetos de aquel estudio, siendo que en el caso de aquellos autores $(n=34)$, la media de edad en los extensionistas salvadoreños estudiados era 41.3 años, contando únicamente con 13.9 años de experiencia laboral. De esa forma, los 22.3 años de experiencia encontrados de los extensionistas de La Libertad y Sonsonate contrastan con los datos encontrados por Landini y Bianqui (2014), ya que la media de años de experiencia laboral reportada para países de América Latina era de 11.1. Este alto índice puede explicarse parcialmente debido a que la media de edad (49.57 años) encontrada en el presente trabajo es superior a la media latinoamericana (40.3 años) reportada por Landini y Bianqui (2014).

Por otra parte, con la excepción de un respondiente, todos los extensionistas del presente estudio tienen formación a nivel superior en temas agropecuarios, ya sea a nivel universitario o técnico (no universitario) ${ }^{3}$. Comparada esta información con los datos reportados para Latinoamérica por Landini y Bianqui (2014), los extensionistas salvadoreños tienen relativamente un bajo nivel de formación, siendo que el nivel de estudios más alto reportado en La Libertad y Sonsonate es grado universitario. En ese sentido, la titulación universitaria más común es el de ingeniería agronómica, que da cuenta del 64.28\% de los casos totales $(n=18)$. Es destacable la ausencia de profesionales de otras áreas comunes en los cuerpos extensionistas como zootecnia, veterinaria o ciencias sociales; identificadas por Landini y Bianqui (2014) para América Latina. Adicionalmente, la mayoría de los extensionistas públicos rurales de estos dos departamentos proceden en su gran mayoría (85.71 \%) de una familia con vocación agropecuaria.

Al ser preguntados sobre cuál es la principal fuente de información en temas agropecuarios, el $60.7 \%$ de ellos respondió que eran los diferentes documentos (investigaciones, manuales, capacitaciones, etc.) emanados de la propia institución pública para la cual ellos trabajan: CENTA ( $\mathrm{n}=17)$. Un $17.9 \%$ respondieron que eran los colegas extensionistas $(n=5)$, mientras que $10.7 \%$ citaron al propio agricultor $(n=3)$ u otra fuente $(10.7 \%)(n=3)$. De esta forma,

\footnotetext{
3 La principal casa de estudios de estos técnicos es la Escuela Nacional de Agricultura (ENA), situada en La Libertad.
} 
ninguno de los respondientes señaló como principal fuente de información en temas agropecuarios a investigaciones científicas, ensayos de campo o revistas especializadas en la materia. Esto se puede deber parcialmente al relativamente bajo nivel de formación de los extensionistas, mencionado anteriormente. De particular importancia es el hecho que un 10.7\% de los extensionistas citan al propio agricultor al cuál ellos están asesorando como la principal fuente de información. Sin desmeritar el conocimiento adquirido por los agricultores a lo largo del tiempo, la ausencia de una base técnica sólida por parte de los extensionistas rurales puede perjudicar la efectividad de la asesoría técnica que ellos proveen. En un sentido similar, Wheeler (2008b) encontró que tener como principal fuente de información sobre temas de agricultura orgánica a medios de comunicación por parte de extensionistas rurales en Australia, estaba asociado a una percepción negativa de esta práctica. Paralelamente, citar a información científica como principal fuente de información sobre orgánicos mostró una percepción positiva sobre esta práctica (Wheeler, 2008b).
Para indagar la percepción de los extensionistas sobre la agricultura orgánica, se utilizó una escala Likert entre 1-5, como lo muestra la tabla 2. Según la información recabada, la mayoría de los respondientes considera que la agricultura convencional es menos amigable con el medio ambiente $(\mu=4.57)$ y es más cara $(\mu=4.21)$ que la agricultura orgánica. Pese a ello, existe una tendencia a considerar que la agricultura convencional permite generar mayores ingresos económicos $(\mu=3.5)$. Asimismo, los respondientes parecen tener un conocimiento modesto sobre agricultura orgánica, ya que la afirmación "poseo amplio conocimiento en relación a la agricultura orgánica" fue puntuada con valor cercanos al $3(\mu=3.2)$, punto medio de la escala Likert implementada.

Según la información cualitativa que se pudo conocer, todo indica que las formaciones de los extensionistas rurales no suelen contener amplio estudio sobre agricultura orgánica. Esto podría justificar a su vez cierta animosidad detectada hacia esta práctica en algunos casos, siendo vista como una forma de agricultura obsoleta. Pese a ello, existe diversa evidencia

\section{Tabla n..$^{\circ}$}

Percepciones de los extensionistas sobre agricultura orgánica

\begin{tabular}{|c|c|c|c|c|c|}
\hline & $\mathbf{N}$ & Min. & Max. & Media & Des. Std. \\
\hline $\begin{array}{l}\text { La agricultura convencional permite generar rendimientos más altos } \\
\text { que la agricultura orgánica }\end{array}$ & 28 & 1 & 5 & 3.50 & 1.232 \\
\hline $\begin{array}{l}\text { La agricultura convencional es menos amigable con el medio } \\
\text { ambiente que la agricultura orgánica. }\end{array}$ & 28 & 1 & 5 & 4.57 & .959 \\
\hline La agricultura convencional es más cara que la agricultura orgánica & 28 & 1 & 5 & 4.21 & 1.134 \\
\hline Poseo amplio conocimiento en relación a la agricultura orgánica & 28 & 1 & 5 & 3.21 & 1.197 \\
\hline
\end{tabular}

Fuente: Elaboración propia en base al trabajo de campo. 
sobre la viabilidad técnica y financiera en la agricultura orgánica frente a la agricultura convencional. En tal sentido Wives (2008) reporta que algunos sistemas productivos en el sur de Brasil son económicamente más eficientes que determinados sistemas convencionales. Para el caso salvadoreño, no fue posible identificar ensayos o investigaciones que compararan la agricultura orgánica con la agricultura convencional, por lo que es probable que las percepciones de estos extensionistas no se basen en una base material, técnica o científica fehaciente. Al no existir mayores investigaciones al respecto, y en virtud que se tiene registro de una producción orgánica mínima a nivel nacional (MINEC, 2009), esta se presenta como una línea de investigación inexplorada en el país.

Dejando de lado la agricultura orgánica, nos centramos en las percepciones de los extensionistas en relación a la cultura de maíz utilizando semilla híbrida y criolla. Como se mencionó anteriormente, la alta utilización de semilla híbrida puede ser explicada parcialmente gracias al largo brazo del apoyo público a este tipo de tecnología. De tal suerte, desde los años dos mil el gobierno ejecutivo dona directamente a pequeños agricultores paquetes agrícolas de maíz y frijol, como forma de promover el cultivo de granos básicos en el país. Los beneficiarios del paquete de apoyo al cultivo de maíz reciben 25 libras $(12.12 \mathrm{Kg})$ de semilla de maíz H-59, y 100 libras (45.45 $\mathrm{Kg}$ ) del abono sulfato de amonio. En tal sentido, se preguntó a los extensionistas el por qué los paquetes gubernamentales cuentan fundamentalmente con semilla de maíz híbrida. E1 $60.7 \%$ considera que el potencial de rendimiento de ese tipo de maíz es superior a otros, el $14.3 \%$ considera que es debido a mayor disponibilidad de semilla $(n=4)$; mientras que el $25 \%$ restante citó otros motivos $(n=7)$. Las respuestas de los extensionistas ya evidencian la importancia del potencial de rendimiento $y$ la disponibilidad de la semilla híbrida. Sobre este último, la investigación doctoral de la cual se desprende este artículo ha podido certificar que en el departamento La Libertad (sede del Centro Nacional de Tecnología Agropecuaria y Forestal y de la Escuela Nacional de Agricultura), los agroservicios solamente ofrecen a la venta semilla de maíz híbrida. De forma paralela, el Estado produce progenitores únicamente de maíz híbrido, sin que exista desarrollo e investigación para maíz criollo. Esto levanta un serio inconveniente a la hora de querer valorar alternativas ambientalmente más limpias, como el cultivo de maíz utilizando semilla criolla, ya que existiría a priori una baja disponibilidad de este insumo. La escasez de semilla criolla es identificada también por Moreno (2000) en Chalatenango, departamento vecino de La Libertad.

Pese a este notable impulso gubernamental a la producción de semilla híbrida de maíz, existe dentro de CENTA un banco de germoplasma que recolecta, cataloga y almacena material genético de una serie de cultivos, incluyendo maíz. Ante la pregunta, ¿por qué motivo existe este Banco que guarda semillas de maíz criollo?, el $42.9 \%$ de los extensionistas consideran que es importante para poder preservar el material genético $(n=12)$. Por su parte, el $28.6 \%$ de los respondientes ven un motivo más utilitario: el resguardo de material genético básico para el fitomejoramiento $(n=8)$. Conservar las variedades nativas de maíz y otros motivos dan cuenta del $14.3 \%$ de las opiniones 
respectivamente $(n=4)$. De esta forma, solamente un pequeño número de respondientes ven útil preservar la variedad de maíz criollo propio de E1 Salvador. Vale la pena destacar que aspectos como el mantenimiento de biodiversidad o el cuido del patrimonio genético autóctono no fueron citados.

Por otra parte, se indagó cuál es la semilla de maíz que recomiendan estos extensionistas a los agricultores asesorados. En una buena parte de los casos, los respondientes expresan una diferencia a la hora de recomendar un tipo de semilla dependiendo de dos condicionantes: la finalidad y las posibilidades económicas del agricultor. En términos generales, cuando el agricultor tiene medios económicos necesarios, los extensionistas recomiendan semilla híbrida que suele ser más cara y tiene requerimientos nutricionales mayores. Por otra parte, cuando el agricultor tiene menor disponibilidad económica, se recomienda una semilla criolla.

Cuando se recomienda semilla híbrida, el tipo más recomendado es $\mathrm{H}-59$ (34.78 \%, $\mathrm{n}=8)$, seguido de H-59 combinado con otro tipo de semilla híbrida en el $30.43 \%$ de los casos $(n=7)$. Igualmente en el $34.78 \%$ los extensionistas recomendaron otro tipo de semilla híbrida $(n=7)$. El argumento más citado para la recomendación de esta semilla es el alto potencial de rendimiento (47.82\%), seguida de la resistencia a plagas en el $21.73 \%$ de los casos $(n=5)$ y buena adaptación en el $17.39 \%$ de las veces $(n=4)$. Nuevamente, la disponibilidad de semilla es citada como motivo para recomendar semilla híbrida en el $8.69 \%$ de los casos $(n=2)$. De esta forma, la disponibilidad de adquisición de semilla híbrida parece confirmarse como una razón de peso en la recomendación de este tipo de insumo. De forma paralela, la semilla H-59 es vista en muchos casos como una semilla propia, en virtud que es subsidiada y producida indirectamente por el propio CENTA.

Por otra parte, cuando se recomienda semilla criolla, el tipo más citado es Santa Rosa en el 40 $\%$ de las veces ( $\mathrm{n}=6$ ), seguido de Capulín en 26.66 $\%$ de los casos $(n=4)$. El restante $33.33 \%$ de los respondientes recomienda otro tipo de maíz criollo. Los motivos para recomendar este tipo de semilla son diversos, entre los que figuran menor costo de adquisición, mejor resistencia a sequías y en general presenta a juicio de estos extensionistas un mejor costo beneficio ante recursos económicos limitados.

Una vez indagados estos aspectos mediante preguntas semi abiertas, se buscó conocer las percepciones de los extensionistas sobre semilla de maíz criolla, particularmente comparándola con la semilla híbrida, misma que es ampliamente utilizada en la agricultura a nivel nacional (MINEC, 2009). El resumen de sus valoraciones está condensado en la tabla 3. Las afirmaciones expuestas para valoración de los extensionistas se centraban en una variedad de características tanto de la semilla de maíz híbrida como criolla, atendiendo a aspectos productivos, adaptativos, nutritivos, económicos y cognitivos.

En relación a aspectos productivos, el que es valorado más positivamente por parte de los extensionistas entrevistados es el potencial de rendimiento del maíz híbrido en relación al maíz criollo $(\mu=4)$. Este suele ser el principal argumento, tanto a nivel público como privado, para la provisión de semilla híbrida de maíz. Este aspecto se ve seguido de la posibilidad 
Tabla n. ${ }^{\circ} 3$

Escala Likert (1-5) reflejando la valoración de los extensionistas en relación a la semilla de maíz híbrida y criolla.

\begin{tabular}{|c|c|c|c|c|c|}
\hline \multicolumn{6}{|c|}{ Percepciones de extensionistas sobre maíz híbrido y criollo } \\
\hline & $\mathbf{N}$ & Min. & Max. & Media & Des. Std. \\
\hline El maíz híbrido tiene mejores rendimientos que el maíz criollo* & 28 & 1 & 5 & 4.00 & 1.186 \\
\hline $\begin{array}{l}\text { El maíz híbrido produce mazorcas y granos de maíz más uniformes } \\
\text { (homogéneos) que el maíz criollo* }\end{array}$ & 28 & 1 & 5 & 3.39 & 1.227 \\
\hline $\begin{array}{l}\text { El maíz híbrido tiene mayor rendimiento para forraje (silo) que el } \\
\text { maíz criollo* }\end{array}$ & 28 & 1 & 5 & 3.25 & 1.378 \\
\hline El maíz híbrido presenta mejor rendimiento de masa que el maíz criollo* & 28 & 1 & 5 & 3.39 & 1.166 \\
\hline $\begin{array}{l}\text { El maíz híbrido es más resistente a sequías, en comparación el } \\
\text { maíz criollo + }\end{array}$ & 28 & 1 & 4 & 2.46 & 1.071 \\
\hline $\begin{array}{l}\text { El maíz híbrido es más resistente a plagas, en comparación el maíz } \\
\text { criollo + }\end{array}$ & 28 & 1 & 5 & 2.54 & 1.138 \\
\hline $\begin{array}{l}\text { El maíz híbrido está mejor adaptado a las condiciones climáticas de } \\
\text { EL SALVADOR que el maíz criollo + }\end{array}$ & 28 & 1 & 5 & 2.32 & 1.219 \\
\hline $\begin{array}{l}\text { El maíz híbrido presenta en términos generales mejores índices en } \\
\text { nutrientes como hierro y zinc que el maíz criollo / }\end{array}$ & 28 & 1 & 5 & 3.04 & 1.319 \\
\hline $\begin{array}{l}\text { El maíz híbrido presenta en términos generales una cantidad de } \\
\text { proteína superior al maíz criollo / }\end{array}$ & 28 & 1 & 5 & 2.50 & 1.171 \\
\hline $\begin{array}{l}\text { El maíz híbrido es preferido por los compradores o intermediarios } \\
\text { de maíz } \$\end{array}$ & 28 & 1 & 5 & 4.07 & 1.120 \\
\hline $\begin{array}{l}\text { El maíz híbrido es preferido por la población en general que compra } \\
\text { y consume maíz \$ }\end{array}$ & 28 & 1 & 5 & 3.36 & 1.224 \\
\hline $\begin{array}{l}\text { El maíz híbrido es más deseado que el maíz criollo por parte de } \\
\text { personas que se dedican a vender o procesar comida (pupusas, } \\
\text { tamales, panaderías, etc.) } \$\end{array}$ & 28 & 1 & 5 & 3.46 & 1.290 \\
\hline $\begin{array}{l}\text { El maíz híbrido es mejor que el maíz criollo para la alimentación } \\
\text { animal \$ }\end{array}$ & 28 & 1 & 5 & 3.00 & 1.277 \\
\hline $\begin{array}{l}\text { La producción de maíz utilizando semilla híbrida es más cara que } \\
\text { utilizando semilla criolla } \$\end{array}$ & 28 & 1 & 5 & 4.36 & .951 \\
\hline $\begin{array}{l}\text { La producción de maíz utilizando semilla híbrida es económicamente } \\
\text { más lucrativo que utilizando semilla criolla } \$\end{array}$ & 28 & 1 & 5 & 3.82 & 1.156 \\
\hline $\begin{array}{l}\text { Las personas que dan asesoría técnica sobre producción de maíz } \\
\text { conocen sobre la producción con semilla criolla \& }\end{array}$ & 28 & 1 & 5 & 3.32 & 1.156 \\
\hline $\begin{array}{l}\text { El maíz híbrido es más conocido que el maíz criollo por parte de los } \\
\text { agricultores \& }\end{array}$ & 28 & 1 & 5 & 3.36 & 1.162 \\
\hline $\begin{array}{l}\text { Durante mi formación en agricultura, era abordada la producción de } \\
\text { maíz con semilla criolla \& }\end{array}$ & 28 & 1 & 5 & 3.32 & 1.362 \\
\hline $\begin{array}{l}\text { Es importante que el Banco de Germoplasma almacene semillas de } \\
\text { maíz criollas originarias de El Salvador }\end{array}$ & 28 & 4 & 5 & 4.86 & .356 \\
\hline Valid N (listwise) & 28 & & & & \\
\hline
\end{tabular}

Símbolos: *: Características productivas. +: Características de adaptación. /: Características nutricionales. \$: Características económicas. \&: Características cognitivas. Fuente: Elaboración propia. 
del maíz criollo de producir mazorcas y granos más uniformes $(\mu=3.39)$ y un rendimiento en masa superior al maíz criollo $(\mu=3.39)$. Siendo que la mayoría del maíz en El Salvador es consumido en forma tortilla, el tema de la producción de masa para la elaboración de ese platillo se vuelve fundamental. Esta valoración sobre el rendimiento del maíz criollo para masa de tortilla contrasta con la opinión de población que consume maíz e incluso de las pequeñas unidades molineras de elaboración de masa, ya que trabajos recientes destacan que diversos actores expresan disconformidad con el desempeño de algunos tipos de maíz híbrido a la hora de elaborar tortillas (Ayala, 2018). En tal sentido, personas dueñas u operarias de molino consideran que el grano del híbrido H-59 es más duro, lo que hace que esos aparatos mecánicos necesiten mayor mantenimiento (Ayala, 2018). Por otra parte, y pese a que estas características productivas son deseadas en la cultura del maíz, los mismos extensionistas reconocen que el maíz híbrido es menos resistente a sequías $(\mu=2.46)$, plagas $(\mu=2.54)$ y que está menos adaptado a las condiciones climáticas del país $(\mu=2.32)$; comparado con el maíz criollo. De tal suerte, bajo la visión de estos extensionistas, pese a que el maíz híbrido tiene mayor potencial de rendimiento, implicaría que la producción tiene mayores riesgos asociados.

En relación a aspectos nutricionales, los extensionistas consideran que el maíz híbrido no tiene mejores índices de proteína que el criollo $(\mu=2.5)$, mientras que no existe una postura clara en relación a nutrientes como hierro y zinc $(\mu=3.04)$. Sobre este particular, es destacable que el mismo CENTA tiene desarrollo, producción y validación de semilla de maíz de alta calidad proteica, como es el caso del maíz híbrido Oro Blanco (CENTA, 2008), Platino (CENTA, 2008) o H-CAS (CENTA, 2016). Pese a ello, los extensionistas no ven en términos generales al maíz híbrido superior al criollo en términos de contenido proteico. Por su parte, existen estudios emanados desde la Escuela Nacional de Agricultura que colocan a maíces criollos salvadoreños con índices similares e incluso superiores a maíces híbridos. De tal suerte en LQA (2015) retrata que las variedades criollas Negrito, Taberón, Olote rosado, Sapo y Chalatenango poseen índices superiores de proteína que el híbrido comercial Dekalb 390. De la misma forma, todos los maíces criollos estudiados en ese trabajo presentaron mejores índices en contenido de hierro que el maíz híbrido Dekalb 390 (LQA, 2015).

En relación a aspectos económicos, existe un entendimiento que la producción con semilla híbrida es más costosa que con semilla criolla ( $\mu=4.36)$. Pese a ello, los extensionistas consideran en buena medida que los compradores o intermediarios agrícolas prefieren el maíz híbrido que su par criollo $(\mu=4.07)$. Esto parece no encontrar total correspondencia con los datos encontrados por Ayala (2018), ya que ese autor retrató que intermediarios agrícolas en La Libertad no tienen preferencia a la hora de comprar (con fines comerciales) maíz. Los mismos expresaban que no acostumbran comprar maíz criollo debido a que no suele haber disponibilidad de este tipo de cereal en particular. Adicionalmente, otros aspectos económicos en orden de importancia decreciente según la media obtenida son: La producción con semilla híbrida es más lucrativa $(\mu=3.82)$, las personas que procesan-venden comida prefieren maíz híbrido $(\mu=3.46)$ y el maíz 
híbrido es preferido por las personas que compran y consumen maíz $(\mu=3.36)$. Nuevamente, estas afirmaciones no encuentran total correspondencia con los datos colectados por Ayala (2018), ya que consumidores y vendedores de maíz consultados no suelen preferir explícitamente maíz híbrido, siendo que en muchos de los casos, los mismos ni siquiera saben el tipo en particular de semilla que los actores consumen o venden. En otras ocasiones, operadores de molino o vendedoras de tortilla han expresado que algunos híbridos como el H-59 producen masa y tortillas mucho más duras, lo que dificulta su procesamiento, consumo $\mathrm{y}$ digestión.

Finalmente, los aspectos cognitivos por los que los extensionistas valoran al maíz híbrido y criollo tienen medias similares. De esa forma, los extensionistas consideran que el maíz híbrido es más conocido que el criollo $(\mu=3.36)$, su formación formal incluía producción de maíz usando semilla criolla $(\mu=3.32)$ y los extensionistas en general conocen sobre producción con semilla criolla $(\mu=3.32)$. Con ello, aparentemente los extensionistas conocen sobre este tema, siendo que las barreras al uso de esta tecnología no serían de orden cognitivo.

\section{Consideraciones finales}

Dado el bajo nivel de investigación en temas de extensión rural en América Latina en general (Méndez, 2006) y en El Salvador en particular, el presente artículo se ha centrado en investigar la percepción de extensionistas rurales públicos en dos departamentos de E1 Salvador: La Libertad y Sonsonate. Se exploran sus percepciones particularmente en relación a agricultura orgánica y semilla de maíz criolla.
Para el caso de la agricultura orgánica, el presente texto ha mostrado que pese a que los extensionistas públicos consideran a esa práctica menos lucrativa que la agricultura convencional, la primera se valora como más amigable con el medio ambiente. De forma adicional, la agricultura orgánica es vista como menos costosa en términos monetarios que la agricultura convencional. Como se ha mencionado, el peso de la agricultura orgánica a nivel nacional es residual (MINEC, 2009), lo que implicaría que beneficios asociados a esa práctica, como aumento de la biodiversidad o menor utilización de insumos, no se vería reflejados en la agricultura nacional. Asimismo, el bajo peso de la agricultura orgánica en el país podría explicar parcialmente la ausencia de estudios que aborden esta práctica agrícola en el país. No obstante, una revisión bibliográfica elaborada por Ayala y Garcez Wives (2018) da cuenta que estudios sobre agricultura orgánica, especialmente enfocada a tomada de decisión, son poco numerosos a nivel mundial y latinoamericano. Especialmente escasos son los estudios que abordan las interrelaciones en temas como desempeño económico, agricultura orgánica, tomada de decisión o extensión rural. Pese a esta relativa carencia, estudios realizados en Brasil han identificado que sistemas productivos orgánicos pueden ser económicamente más eficientes que sistemas convencionales (Wives, 2008). De tal suerte, y en función de la ausencia de este tipo de trabajos en El Salvador, estos temas se presentan como una línea de investigación fecunda e inexplorada.

Por otra parte, las percepciones de los extensionistas rurales sobre la cultura de maíz utilizando semilla criolla e híbrida presenta resultados difusos. De esa forma, los 
extensionistas consideran que la producción con semilla híbrida tiene mayor potencial de rendimiento y de producir mazorcas con granos más uniformes; si bien reconocen que es más cara y más vulnerable a plagas y sequías. De forma adicional, los extensionistas consideran que la semilla híbrida es más deseada por consumidores e intermediarios de maíz, si bien la evidencia disponible apunta en otra dirección. En cualquier caso, algunas de estas valoraciones parecen no estar cimentados sobre una base técnica científica en virtud de al menos dos motivos: a) No existen amplios estudios sobre producción de maíz con semilla criolla en el país $y$, b) la principal fuente de información en temas agropecuarios para el $60.7 \%$ de los respondientes es el propio CENTA, organismo que no realiza a priori investigación y desarrollo de maíz criollo. Como se ha mencionado, únicamente fue posible identificar algunos esfuerzos a nivel privado por temas relacionados al maíz criollo (MORENO, 2000; CORDES, 2016; RIVAS et al.; 2013).

Centrarse en una de las dimensiones que componen los sistemas agroalimentarios localizados, específicamente en la dimensión institucional (Muchnik, 2006), ha permitido aproximarse al papel que los extensionistas rurales tienen en el proceso de asesoramiento a agricultores, así como en la difusión de tecnología. Dado estos resultados, la evidencia indicaría a que los extensionistas rurales en Sonsonate y La Libertad presentarían una tendencia hacia la recomendación en la utilización de semilla de maíz híbrida. No obstante, dado que estos profesionales toman en consideración aspectos productivos, económicos, adaptativos, cognitivos y nutricionales a la hora de recomendar la utilización de un tipo de semilla de maíz; se necesitan estudios más amplios que puedan determinar y cuantificar qué aspectos concretos influencian la recomendación de semilla criolla e híbrida en el país. En virtud que las agencias públicas responsables por temas agrícolas y su personal son la principal fuente de información para agricultores (Ban y Hawkings, 1988), se vuelve fundamental poder identificar y cuantificar qué aspectos influencian la recomendación en uso de semilla criolla, particularmente si se pretenden implementar iniciativas más responsables en términos medioambientales.

\section{Bibliografía}

Ambrosini, L.; Filippi,E. y Miguel, L. (2008). Sial: Analise da produção agroalimentar a partir de um aporte territorialista e multidisciplinar. Ideas: Interfaces em desenvolvimento, agricultura e sociedade, 2(1), 6-31.

Ambrosini, L; Filippi,E. y Miguel, L. (2009). Produção de queijo serrano: Estratégia de reprodução social dos pecuaristas familiares do sul do Brasil sob a perspectiva muldisciplinar do sistema agroalimentar localizado - sial. Estudo E̋ Debate, 16 (2), 27-54.

Ardila, J. (2010). Extensión rural para el desarrollo de la agricultura y la seguridad alimentaria: aspectos conceptuales, situación y una visión de futuro. San José, Costa Rica: Instituto Interamericano de Cooperación para la Agricultura.

Asociación Fundación para la Cooperación y el Desarrollo Comunal de El Salvador-CORDES. (2016). Técnicas de Producción, Conservación Bancos de Semillas Criollas. San Salvador: CORDES- Centro de Estudios Rurales y Agricultura Internacional- Unión Europea. 
Ayala Durán, C. (2018). Sistema agroalimentario del maíz blanco en Ciudad Arce, municipio de E1 Salvador, tesis doctoral, Universidade Federal do Rio Grande do Sul, Porto Alegre, Brasil.

Ayala, C., Garcez Wives, D. (2018). Decision Making and Agriculture: A recent Review of Organic Farming. Desenvolvimento em Questão, 16 (43), 175-199.

BANCO CENTRAL DE RESERVA -BCR. (2008). Boletín Económico. La Economía Nacional y de los Hogares Salvadoreños: Impacto de los Precios de los Alimentos y del Petróleo. San Salvador: BCR.

Bengtsson, J.; Ahnström, J.; y Weibull, A. (2005). The effects of organic agriculture on biodiversity and abundance: a meta-analysis. Journal of Applied Ecology, 42 (2), 261-269. doi: 10.1111/j.1365-2664.2005.01005.x.

CENTRE DE COOPÉRATION INTERNATIONALE EN RECHERCHE AGRONOMIQUE POUR LE DÉVELOPPEMENT -CIRAD. (1996) Systèmes Agroalimentaires Localisés: organisations, innovations et developpement local orientations et perspectives issues de la consultation du CIRAD. Stratégies de recherche dans le domaine de la socio-économie de l'alimentation et des industries agroalimentaires. Paris: CIRAD-SAR, 1996.

CENTRO NACIONAL DE TECNOLOGÍA AGROPECUARIA Y FLORESTAL CENTA. (2008). Híbridos de maíz de alta calidad protéica Oro Blanco y Platino. Boletín Térnico 20. San Andrés, E1 Salvador: CENTA.
CENTRO NACIONAL DE TECNOLOGÍA AGROPECUARIA Y FORESTAL -CENTA. (2016). CENTA H-CAS. Hibrido de maiz de alta calidad proteica para nutrir a El Salvador. San Andrés, E1 Salvador: CENTA.

\section{CENTRO NACIONAL DE TECNOLOGÍA} AGROPECUARIA Y FORESTAL- CENTA. (2015). PROYECTO: "Biofertilizantes en Cultivo de Maiz, Frijol y café como Alternativa Agroecológica para una Producción Sostenible en El Salvador". San Andrés, El Salvador: CENTA.

Chang, H., y Kristiansen, P. (2006). Selling Australia as clean and green. Australian Journal of Agricultural E Resource Economics, 50, 103113. doi: https://doi.org/10.1111/j.14678489.2006.00330.x.

Chechi, L.; Schultz, G.; Niederle, P. (2016). Tradição e inovação entrelaçadas na consolidação de um Sistema Agroalimentar Localizado de erva-mate no sul do Brasil. Eutopia, 10, 79-93.

Cortez, M.J. (2014). Fitomejoramiento de maices criollos utilizando la técnica de selección masal estratificada como apoyo al productor indigena salvadoreño. La Libertad: Escuela Nacional de Agricultura.

Duram, L. y Larson, K. (2001). Agricultural research and alternative farmers information needs. Professional Geographer, 53 (1),84-96. doi: 10.1080/00330124.2001.9628438.

Escobar, G. (2012). Diseño de una agenda de extensión rural latinoamericana para un desarrollo rural inclusivo. Extensión rural con un enfoque participativo y de mercado: hacia un marco 
conceptual. Santiago: Centro Latinoamericano para el Desarrollo Rural.

FUNDACIÓN SALVADOREÑA PARA LA RECONSTRUCCIÓNYELDESARROLLO -REDES. (2014). La agricultura familiar en el centro de las políticas agrícolas. Análisis del Plan de Agricultura Familiar (PAF) y su presupuesto. El Salvador, 2011-2014. San Salvador: REDES.

T. Gomiero , M.; Paoletti, G. y Pimentel, D. (2008). Energy and Environmental Issues in Organic and Conventional Agriculture, Critical Reviews in Plant Sciences, 27:4, 239-254. DOI: $10.1080 / 07352680802225456$.

Goodell, G.; Andrews, K.; y López, J. (1990). The contributions of agronomoanthropologists to on-farm research and extension in integrated pest management. Agricultural Systems, 32(4), 321-340. doi: 10.1016/0308-521X(90)90098-B.

Grass-Ramírez, J.; Cervantes-Escoto, F. y Palacios-Rangel, M. (2016). ELEMENTOS METODOLÓGICOS PARA EL FORTALECIMIENTO DEL ENFOQUE DE SISTEMAS AGROALIMENTARIOS LOCALIZADOS (SIAL). Agricultura, Sociedad y Desarrollo, 13(1), 63-85.

Gravina, V. (2016). Percepciones sobre el liderazgo de los extensionistas rurales en Uruguay, un abordaje cuantitativo. Agrociencia Uruguay, 20(2), 145-152.

Hötzel, M.K., y Sneddon, J.N. (2013). The role of extensionists in Santa Catarina, Brazil, in the adoption and rejection of providing pain relief to calves for dehorning. Journal of Dairy Science, 96(3), 1535-1548. doi: 10.3168/jds.2012-5780.

\section{LABORATORIO DE QUÍMICA} AGRÍCOLA -LQA. (2015) Análisis bromatológico: Porcentaje de proteina, promedio de hierro y zinc (partes por millón). San Andrés, La Libertad: Escuela Nacional de Agricultura.

Landini, F. (2012). Problemas en la extensión rural paraguaya: modelos de extensión en la encrucijada. Cuadernos de desarrollo rural, 9 (69), 127-149.

Landini, F. (2015). Problemas enfrentados por extensionistas rurais brasileiros e sua relação com suas concepções de extensão rural. Ciência Rural, 45(2), 371-377. https://dx.doi. org/10.1590/0103-8478cr20140598.

Landini, F. (2015b). Problemas percibidos y concepciones de extensión de los técnicos del Centro Nacional de Tecnología Agropecuaria y Forestal de El Salvador, Centroamérica. INTERAÇÕES, 16(2), 455-464. doi: 10.1590/151870122015219.

Landini, F. (2016). Diversidad de expectativas de los extensionistas rurales latinoamericanos sobre la psicología. Estudos de Psicologia, 21(4), 392402. doi: 10.5935/1678-4669.20160038.

Landini,F.(2016b).Enfoques y prácticas de extensión rural públicas en el noreste argentino. Revista de Economia e Sociologia Rural. 54(1), 167-186. doi: 10.1590/1234-56781806-9479005401009.

Landini, F. (2016c). Problemas de la extensión rural en América Latina. Perfiles Latinoamericanos, 24(47), 47-68. doi:10.18504/p12447-005-2016. 
Landini, F.(2016d). Unfolding the knowledge and power dynamics of the 'farmers-rural extensionists' interface in North-Eastern Argentina. The Journal ofAgricultural Education and Extension, 22(5), 1-15. doi: 10.1080/1389224X.2016.1227050.

Landini, F., y Bianqui, V. (2014). Sociodemographic profile of different samples of Latin American rural extensionists. Ciência Rural, 44(3), 575-581. doi: 10.1590/S010384782014000300030 .

Landini, F., y Bianqui, V. (2014b). La extensión rural en Ecuador desde el punto de vista de sus extensionistas. Revista de la Facultad de Agronomia, 31 (3), 433- 454.

Landini, F.; Bianqui, V.; y Crespi, M. (2013). "Evaluación de las creencias sobre extensión rural de los extensionistas paraguayos". PSIENCIA. Revista Latinoamericana de Ciencia Psicológica, 5(1) 3-14. doi: 10.5872/psiencia/5.1.21.

Landini, F., y Riet, L. (2015). Extensión rural en Uruguay: problemas y enfoques vistos por sus extensionistas. Mundo agrario, 16(32), 1-17.

Larson, K. L., y Duram, L. A. (2000). Information dissemination in alternative agriculture research: An analysis of researchers in the north central region. American Journal of Alternative Agriculture, 15(4), 171-180. doi: 10.1017/S0889189300008742.

Mäder, P.; Fliebach, A.; Dubois, D.; Gunst, L.; Fried, P., y Niggli, U.(2002).Soil fertility and biodiversity in organic farming. Science, 296 (5573), 1694-1697. doi:10.1126/ science.1071148.
Magdaleno-Hernández, E.; Mejía-Contreras, A.; Martínez-Saldaña, T.; Jiménez-Velazquez, M.; Sanchez-Escudero, J.; y García-Cué, J. (2016). Selección tradicional de semilla de maíz criollo. Agricultura, sociedad y desarrollo, 13(3), 437-447.

Malafaia, G. C. y Barcellos, J. O. J. (2007). Sistemas Agroalimentares Locais e a Visão Baseada em Recursos: construindo vantagens competitivas para a carne bovina gaúcha. Revista de Economia e Agronegócio (5), 25-50.

Martínez, G. (2007). Competitividad agrícola en Centroamérica. Implicaciones de las normas de propiedad intelectual contenidas en el CAFTA$D R$ con relación al mercado de agroquímicos. San Salvador: FUNDE.

Méndez, M. (2006). Los retos de la extensión ante una nueva y cambiante noción de lo rural. Revista Facultad Nacional de Agronomía, 59(2), 3407-3423. doi: 10.15446/rfnam.

Ministerio de Agricultura y Ganadería -MAG. (2016, Julio, 7). MAG beneficia a centenares de productores de hortalizas con casas malla. Noticias del Ministerio de Agricultura y Ganadería. Consultado en:www.mag.gob.sv/mag-beneficiaa-centenares-de-productores-de-hortalizascon-casas-malla/.

Ministerio de Economía de El SalvadorMINEC-. (2009). IV Censo Agropecuario 20072008. Resultados Nacionales. San Salvador: MINEC.

Moreno, S. (2000, Diciembre, 26). El maíz criollo se niega a desaparecer. El Diario de Hoy. 
Consultado en: http://archivo.elsalvador.com/ noticias/EDICIONESANTERIORES/2000/ DICIEMBRE/diciembre26/ELPAIS/elpais1. html.

Muchnik, J. (2006). Sistemas Agroalimentarios Localizados: evolución del concepto y diversidad de situaciones. In: CONGRESO INTERNACIONAL DE LA RED SIAL: Alimentación y Territorios, 3, 2006, Baeza España. Anales. Baeza, 2006.CD-ROM.

Muchnik, J. (2012). Sistemas agroalimentarios localizados: desarrollo conceptual y diversidad de situaciones. In: SALCIDO, G.; TORES, R.M. Los sistemas agroalimentarios localizados. Identidad territorial, construcción de capital social e instituciones. Ciudad de México: UNAM. pp. 42-52.

Nelson, L.; Giles, J.; Macilwain, C. y Gewin, V. (2004). Organic FAQs. Nature. 428, 796-798. DOI:10.1038/428796a.

Organización de las Naciones Unidas para la Alimentación y la Agricultura - FAO (2004). The scope of organic agriculture, sustainable forest management and ecoforestry in protected area management. Working Paper No. 18. Roma: FAO.

O'Riordan, T., y Cobb, D.(2001). Assessing the consequences of converting to organic agriculture. Journal of Agricultural Economics, 52 (1), 22-35. doi: 10.1111/j.1477-9552.2001.tb00907.x.

Rivas, G., Rodríguez; A., Padilla, D.; Hernández, L. y Suchini, J. (2013) Bancos Comunitarios de Semillas Criollas: Una opción para la conservación de la agrobiodiversidad. Turrialba, Costa Rica: Centro Agronómico Tropical de Investigación y Enseñanza.
Specht, S. (2009). O território do morango no Vale do Cai - RS: análise pela perspectiva dossistemas agroalimentares localizados. Tese de Doutorado. Programa de Pós Graduação em Desenvolvimento Rural. Universidade Federal do Rio Grande do Sul. Porto Alegre.

Specht, S. (2014). MORANGOS DO VALE DO CAÍ-RS: um sistema agroalimentar territorializado. CAMPO TERRITÓRIO, 9 (19), 6-31.

Stolze,M.; Piorr, A.; Haring, A. y Dabbert, S. (2000). The environmental impacts of organic farming in Europe. Organic Farming in Europe: Economics and Policy, vol. 6. Hohenheim, Ger: University of Hohenheim.

United States Department of AgricultureSecretaria de Agricultura Ganadería y Desarrollo Rural [USDA-SAGAR] (1997). Situación actual y perspectiva de la producción de maíz en México 1990-1997. Ciudad de México: Departamento de Agricultura de los Estados Unidos y la Secretaría de Agricultura y Desarrollo Rural.

Van den Ban, A., y Hawkins, H. (1988). Agricultural Extension. Essex: Longman Scientific and Technical.

Wheeler, S. (2008a). Exploring professional attitudes towards organic farming, genetic engineering, agricultural sustainability and research issues in Australia. Journal of Organic Systems, 3(1), 37-56.

Wheeler,S.(2008b). What influences agricultural professionals' views towards organic agriculture?. Ecological Economics, 65(1), 145-154. http:// dx.doi.org/10.1016/j.ecolecon.2007.05.014. 
Wives, D.G. (2008). Funcionamento eperformance dos sistemas de produção da banana na microrregião do Litoral Norte do Rio Grande do Sul. (Master dissertation).

Wynen, E. (1989). Sustainable and conventional agriculture in South-Eastern Australia: A comparison. (Doctoral Dissertation).
Zilli, I. ; Bregt, A. y Rossiter, D. (2006). A participatory approach for integrating risk assessment into rural decision-making: A case study in Santa Catarina, Brazil. Agricultural Systems, 87(2), 229-244. doi: 10.1016/j. agsy.2005.01.008. 